\title{
Combination immunotherapy with anti-VEGF/TKI for hepatocellular carcinoma: present and future perspective
}

\author{
Masatoshi Kudo \\ Department of Gastroenterology and Hepatology, Kindai University Faculty of Medicine, Osaka, 589-8511, Japan \\ Correspondence to: Masatoshi Kudo, MD, PhD. Department of Gastroenterology and Hepatology, Kindai University Faculty of Medicine, 377-2, \\ Ohno-Higashi, Osaka-Sayama, Osaka, 589-8511, Japan. Email: m-kudo@med.kindai.ac.jp.
}

Submitted Sep 12, 2020. Accepted for publication Sep 23, 2020.

doi: $10.21037 / \mathrm{hbsn}-20-707$

View this article at: http://dx.doi.org/10.21037/hbsn-20-707

The results of the Phase 3 IMbrave150 trial were published in the New England Fournal of Medicine (1). The IMbrave150 trial is a global multicenter phase 3 comparative study conducted in patients with locally advanced or metastatic liver cancer and unresectable hepatocellular carcinoma (HCC). Patients were assigned, 2:1, to atezolizumab plus bevacizumab (Atezo + Beva) $(n=336)$ and sorafenib $(n=165)$ groups. The co-primary endpoints were progressionfree survival (PFS) and overall survival (OS) using the independent review facility (IRF)-assessed Response Evaluation Criteria in Solid Tumors (RECIST) v1.1. Stratification factors were geographic region (Asia excluding Japan vs. rest of world), Eastern Cooperative Oncology Group (ECOG) performance status (PS) (0 vs. 1), macrovascular invasion (MVI) and/or extrahepatic spread (EHS) (presence $v s$. absence), and serum alphafetoprotein (AFP) level ( $\leq 400 v s$. $>400 \mathrm{ng} / \mathrm{mL}$ ). The proportion of patients with MVI was $38 \%$ (129/336), MVI and/or EHS was $78 \%$ (258/336), and AFP $\geq 400 \mathrm{ng} / \mathrm{mL}$ was $38 \%(126 / 336)$ in the IMbrave150 population and their prognosis was poor. Nevertheless, surprisingly good results were observed in the IMbrave 150 trial. The OS in the Atezo + Beva group was not estimable (NE) and that in the sorafenib group was 13.2 months [95\% confidence interval (CI), 10.4-NE]. Additionally, a surprising hazard ratio, of 0.58 (95\% CI, 0.42-0.79, P=0.0006) was observed. It is worth noting that the $\alpha$ given to the OS for the $1^{\text {st }}$ interim analysis of this trial was 0.0033 and the results in this study sufficiently cleared this value (1).

The PFS, by IRF-assessed RECIST $\mathrm{v} 1.1$, in the IMbrave 150 trial was 6.8 months (95\% CI, 5.7-8.3) in the Atezo + Beva group and 4.3 months (95\% CI, 4.0-5.6) in the sorafenib group and the HR was 0.59 (95\% CI, 0.47-0.76, $\mathrm{P}<0.0001)$. Although the Phase 1b Arm A (2) PFS value was lower at 7.3 months, this was considerably longer than the 5.6 months observed in Arm F (2). As the observation period for IMbrave150 (8.6 months) was shorter than that for Arm A (12.4 months) an extension of the observation period may result in a greater PFS.

The objective response rate (ORR), by IRF-assessed RECIST v1.1, was significantly higher in the Atezo + Beva group $(27 \%$; 95\% CI, 23-33) than in the sorafenib group (12\%; 95\% CI, 7-18) (stratified P value $<0.0001$ ). The ORR in the Atezo + Beva group was superior in Phase $1 \mathrm{~b}$ Arm A (36\%) than in Arm F (20\%), and this may be further improved in the final analysis, in which the observation period is extended. A complete response was observed in 18 patients $(6 \%)$, which was a promising result. The median duration of response (DOR) was NE and DOR $>6$ months was observed in $80 \%$ of responders, which indicated a durable response.

A more favorable patient-reported outcome was observed in the Atezo + Beva group than in the sorafenib group. The decrease in quality of life (QOL) in the Atezo + Beva group was more favorable than that in the sorafenib group, with an HR of 0.63 (95\% CI, 0.46-0.85, $\mathrm{P}=0.0028$ ), which is comparable to that reported for a similar antibody, ramucirumab (3), and this cannot be achieved with tyrosine kinase inhibitors (TKIs), which induce adverse events (AEs). For example, time to symptomatic progression was examined in the SHARP trial but no improvements were observed. These favorable results observed in IMbrave150 may be caused by the delayed onset of symptoms owing to the drug and as this combination is an antibody-based 
drugs, which cause minimal AEs and is well tolerated. A subset-analysis in IMbrave150 showed that the Atezo + Beva group was superior in both OS and PFS than the sorafenib group in all subsets except intermediate stage HCC.

In conclusion, Atezo + Beva produced promising results, outperforming sorafenib in all endpoints, OS, PFS, ORR, and safety, and categories in the planned interim analysis (OS, PFS by IRF-assessed RECIST v1.1, ORR by IRF RECIST v1.1, and ORR by IRF-assessed mRECIST v1.1) (1). This can be said to be an important result, in which the theory that proposes that enhanced programmed death-ligand 1 (PD-L1) antibody effects are induced by anti-vascular endothelial growth factor (VEGF) antitumor effects by changing the immune microenvironment from suppressive to responsive was supported in clinical trials $(4,5)$. This Atezo + Beva combination is the only drug that has shown superiority compared with sorafenib since 2007 and, in this sense, is a landmark result. Surprisingly, cancer and the immune system responded as suggested by this theory. Thus, this could be a milestone in the development of systemic therapy for HCC $(1,4,5)$.

The immune class of HCCs reported by Sia et al. (6) is important for understanding differences between a programmed cell death protein 1 (PD-1)/PD-L1 antibody monotherapy and PD-1/PD-L1 antibody plus anti-VEGF antibody/TKI combination therapy. Sia $e t$ al. showed that approximately $30 \%$ of all patients with HCC have immune hot tumors with lymphocyte infiltration, in which there is an abundance of interferon- $\gamma$, granzymes, immune cells, and PD-L1; therefore, immune checkpoint inhibitors (ICIs) may be effective. This corresponds to the Type I and IV tumor microenvironments (TMEs) reported by Teng et al. (7). ICI monotherapy is effective in $20 \%$ of the active immune class group, which corresponds to the Type I TME. However, $10 \%$ of the exhausted immune class is in a state in which T-cell activity is suppressed (i.e., immune exhausted) by various suppressive TMEs, which corresponds to the Type IV TME. Therefore, in Type IV TMEs, ICI monotherapy may not be effective. However, although effects may not be expected from PD-1/PD-L1 antibody monotherapy for tumors with an immunosuppressive TME (i.e., immune exhausted subclass), it is estimated that PD-1/ PD-L1 antibodies will activate CD8-positive cells as a result of anti-VEGF antibody/TKI therapy by improving the TME. Furthermore, the $\mathrm{Wnt} / \beta$-catenin mutation is an activating mutation, in which $\beta$-catenin is activated, with a frequency of approximately $20-30 \%$. Sia et al. classified
HCCs containing this $W n t / \beta$-catenin mutation as the immune exclusion class and proposed that it is resistant to ICI treatment (i.e., primary resistance) (6).

PD-1/PD-L1 monotherapy only exerts (I) an inhibitory effect on the PD-1/PD-L1 pathway, and effects of the tumor, with immunosuppressive TME or $\beta$-catenin mutation cannot be expected. In contrast, in addition to inhibiting the PD-1/PD-L1 pathway, PD-1/PD-L1 antibody plus anti-VEGF/TKI combination therapies, for example, using bevacizumab or TKIs, may exert combined effects such as: (II) inhibiting the VEGF-A signaling pathway and killing the tumor cell irrespective of $\beta$-catenin mutation, (III) increasing cancer antigen release by HCC necrosis, (IV) activating the maturation of dendritic cells by anti-VEGF antibodies and improving the ability to recognize and present cancer antigens, $(\mathrm{V})$ improving the ability to activate CD8-positive cells at the priming phase, (VI) increasing tumor infiltration by CD8-positive cells by normalizing abnormal tumor vessels (owing to the action of anti-VEGF antibodies), and (VII) improving the suppressive TME (owing to anti-VEGF effects). Thus, anti-PD-1/PD-L1 antibody plus anti-VEGF antibody/ TKI combination therapy, with the inhibition of the PD-1/ PD-L1 pathway, produces synergistic effects by acting in an immunostimulatory manner in every step of the cancer immunity cycle (Table 1) as a result of the (I) release of cancer antigens, (II) enhanced ability of dendritic cells to present cancer antigens, (III) antigen-specific T cell activation by enhanced priming capacity in lymph nodes, and (IV) stimulation of CD8-positive cell infiltration into the tumor, after which activated $\mathrm{T}$ cells attack cancer cells owing to the anti-PD-1/PD-L1 pathway blockade and improvement in the immunosuppressive TME.

The release of VEGF-A from the hypoxic tumor increases the number of suppressive molecules, such as tumor-associated macrophages, regulatory $\mathrm{T}$ cells, and myeloid-derived suppressor cells (8). Furthermore, immunosuppressive cytokines interleukin-10 and transforming growth factor- $\beta$ are released to further suppress the TME. This inhibits the maturation of dendritic cells and the activation/proliferation of T-cells. However, the administration of anti-VEGF antibodies improves the immunosuppressive environment and T-cell activation/maturation by dendritic cells. Therefore, antiVEGF antibodies exert their effects and contribute to the restoration of CD8-positive cell immune activity when CD8-positive activation is suppressed by the TME, even 
Table 1 Synergistic effect of combination immunotherapy with PD-1/PD-L1 antibody and anti-VEGF/TKI

\begin{tabular}{lll}
\hline & PD-1/PD-L1 antibody monotherapy & PD-1/PD-L1 antibody plus anti-VEGF/TKI combination immunotherapy \\
\hline Mode of action & PD-1·PD-L1 blockade & PD-1·PD-L1 blockade \\
& Direct antitumor effect by inhibiting signaling pathway \\
& Increased cancer antigen release by tumor necrosis \\
& Increased antigen presentation by maturation of dendritic cell \\
& Increased activation of CD8+ T cell at the priming phase \\
& Increased tumor infiltration of CD8+ T cell by normalization of abnormal tumor \\
& vessel \\
& Improvement of tumor microenvironment from immune suppressive to immune \\
& responsive
\end{tabular}

VEGF, vascular endothelial growth factor; PD-1, programmed cell death 1; PD-L1, programmed cell death ligand 1; TKI, tyrosine kinase inhibitor.

when CD8-positive cells infiltrate in the cancer, as in Type IV tumors.

In addition to the low immunogenicity of TMEs in Type II tumors, Type II cases include cancers with Wnt/ $\beta$-catenin-activating mutations, as proposed by Llovet et al.; therefore, the Type II TME does not appear to be infiltrated by CD8-positive cells. As such, antitumor effects may be exerted by the direct antitumor and necrotic effects of HCCs by the anti-VEGF antibodies/TKIs themselves, even in Type II tumors, which do not originally have immune cell infiltration. Furthermore, CD8-positive cell infiltration and the exertion of ICI effects may be caused by the increased immunogenicity induced by necrosis. Among the clinical cases in Harding et al. (9), All of 10 patients with Wnt/ $\beta$-catenin mutations had progressive disease (PD), whereas 17 patients without $\mathrm{WNT} / \beta$-catenin mutation had a disease control rate of $71 \%$ (9). Furthermore, the PFS in patients with the $\mathrm{Wnt} / \beta$-catenin mutation administered ICI monotherapy was 2.0 months, whereas the PFS in patients without this mutation reached 7.4 months $(\mathrm{P}<0.0001)$. The disease control rate value of $71 \%$ is in good agreement with the value of approximately $70 \%$ when the immune hot subclass $(30 \%)$ and immune moderate subclass $(40 \%)$ are combined. However, There was no relationship between effects of sorafenib and the presence or absence of Wnt/ $\beta$-catenin mutations (9).

The $\mathrm{Wnt} / \beta$-catenin mutation was originally considered to be present in $20-30 \%$ of all HCCs. Indeed, PD rates from single-agent nivolumab, single-agent pembrolizumab, and single-agent atezolizumab are as high as $37 \%, 32.4 \%$, and $42.0 \%$, respectively $(2,10,11)$ (Table 2$)$. However, combined PD-1/PD-L1 and anti-VEGF/TKI therapy reduces $\mathrm{PD}$ rates to $20 \%$ for the Atezo + Beva group (Phase 3 IMbrave150) (1), 28\% for the Atezo + Beva group (Phase $1 \mathrm{~b}$ Arm F), $24.0 \%$ for the Atezo + Beva group (Phase 1b Arm A) (2), 7.0\% for the pembrolizumab + lenvatinib group (Phase 1b) (12), and $8.3 \%$ for the nivolumab + lenvatinib group (Phase 1b) (13) (Table 2). The ORR of bevacizumab is $13 \%$ in RECIST v1.0, whereas the ORR of lenvatinib is $18.8 \%$ in RECIST v1.1 and 40.6\% in mRECIST, indicating a strong antitumor effect of lenvatinib (14). This may result in a lower PD rate because multikinase inhibitors, such as lenvatinib, have higher tumor-necrosis and cancerantigen release effects than bevacizumab; therefore, the combination of lenvatinib and pembrolizumab exerts a greater anti-tumor effect on Type IV and probably Type II HCC than each drug alone (15). These combination therapies with ICIs and anti-VEGF antibody/TKIs may be effective in tumors with $\mathrm{Wnt} / \beta$-catenin activating mutations such as Type II HCC. As a result, lower PD rates have led to prolonged PFS (Table 2).

These results suggest that combination immunotherapy would be more effective than ICI monotherapy owing to the synergistic effects brought about by: (I) anti-VEGF activity (improved TME, dendritic cell activation, and immune cell infiltration into the tumor), (II) direct anticancer activity (increased release of cancer antigens by a blockade of VEGF and multi-kinase pathways), (III) blockade of the PD-1/ PD-L1 pathway (activation of CD8-positive cells), and (IV) effects on $W n t / \beta$-catenin activated mutations.

A Phase 3 LEAP002 trial, which compares pembrolizumab + lenvatinib combination therapy with 


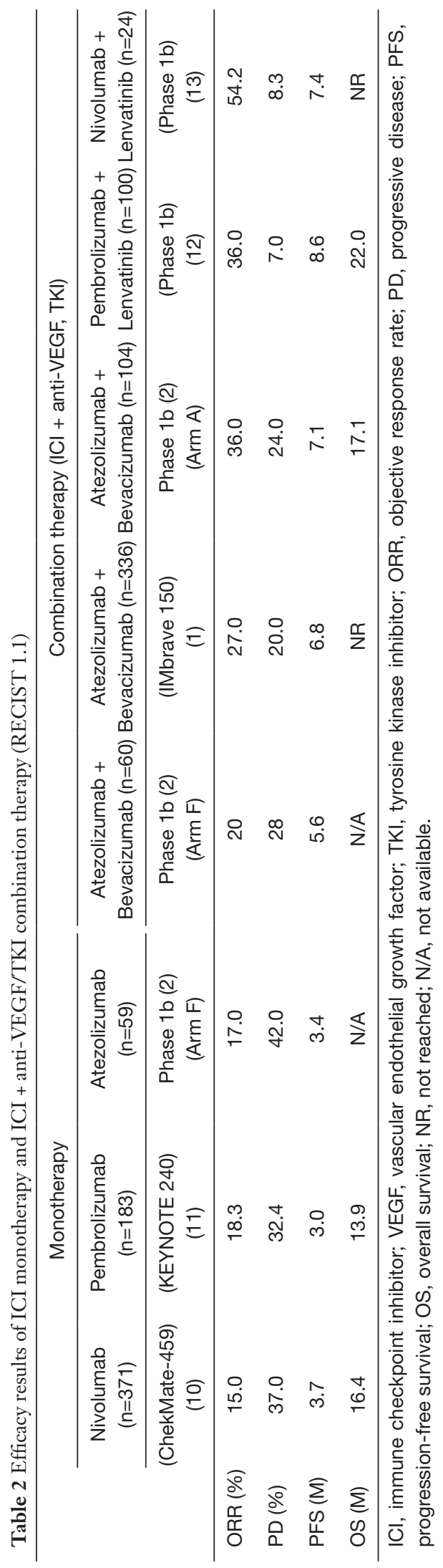

lenvatinib alone, is in progress and its results are greatly awaited.

\section{Acknowledgments}

Funding: None.

\section{Footnote}

Provenance and Peer Review: This article was commissioned by the editorial office, Hepatobiliary Surgery and Nutrition. The article did not undergo external peer review.

Conflicts of Interest: The author has completed the ICMJE uniform disclosure form (available at available at https:// hbsn.amegroups.com/article/view/10.21037/hbsn-20-707/coif). Dr. MK reports personal fees from Bayer, grants, personal fees and other from Eisai, personal fees and other from MSD, grants from Otsuka, grants from Taiho, grants from EA Pharma, grants from Takeda, grants from Gilead, grants from Abbvie, grants and other from Ono, personal fees and other from Bristol-Myers Squibb, grants from Sumitomo Dainippon Pharma, personal fees from Lilly, other from Roche, outside the submitted work.

Ethical Statement: The author is accountable for all aspects of the work in ensuring that questions related to the accuracy or integrity of any part of the work are appropriately investigated and resolved.

Open Access Statement: This is an Open Access article distributed in accordance with the Creative Commons Attribution-NonCommercial-NoDerivs 4.0 International License (CC BY-NC-ND 4.0), which permits the noncommercial replication and distribution of the article with the strict proviso that no changes or edits are made and the original work is properly cited (including links to both the formal publication through the relevant DOI and the license). See: https://creativecommons.org/licenses/by-nc-nd/4.0/.

\section{References}

1. Finn RS, Qin S, Ikeda M, et al. Atezolizumab plus Bevacizumab in Unresectable Hepatocellular Carcinoma. N Engl J Med 2020;382:1894-905.

2. Lee MS, Ryoo BY, Hsu CH, et al. Atezolizumab with or without bevacizumab in unresectable hepatocellular carcinoma (GO30140): an open-label, multicentre, phase 
1b study. Lancet Oncol 2020;21:808-20.

3. Zhu AX, Kang YK, Yen CJ, et al. Ramucirumab after sorafenib in patients with advanced hepatocellular carcinoma and increased alpha-fetoprotein concentrations (REACH-2): a randomised, double-blind, placebocontrolled, phase 3 trial. Lancet Oncol 2019;20:282-96.

4. Kudo M. Immuno-Oncology Therapy for Hepatocellular Carcinoma: Current Status and Ongoing Trials. Liver Cancer 2019;8:221-38.

5. Kudo M. Combination Cancer Immunotherapy with Molecular Targeted Agents/Anti-CTLA-4 Antibody for Hepatocellular Carcinoma. Liver Cancer 2019;8:1-11.

6. Sia D, Jiao Y, Martinez-Quetglas I, et al. Identification of an Immune-specific Class of Hepatocellular Carcinoma, Based on Molecular Features. Gastroenterology 2017;153:812-26.

7. Teng MW, Ngiow SF, Ribas A, et al. Classifying Cancers Based on T-cell Infiltration and PD-L1. Cancer Res 2015;75:2139-45.

8. Voron T, Marcheteau E, Pernot S, et al. Control of the immune response by pro-angiogenic factors. Front Oncol 2014;4:70.

9. Harding JJ, Nandakumar S, Armenia J, et al. Prospective Genotyping of Hepatocellular Carcinoma: Clinical Implications of Next-Generation Sequencing for Matching
Patients to Targeted and Immune Therapies. Clin Cancer Res 2019;25:2116-26.

10. Yau T, Park JW, Finn RS, et al. CheckMate 459: A randomized, multi-center phase III study of nivolumab vs sorafenib as first-line treatment in patients with advanced hepatocellular carcinoma. Ann Oncol 2019;30:v874-v875.

11. Finn RS, Ryoo BY, Merle P, et al. Pembrolizumab (Pembro) therapy vs best supportive care (BSC) in advanced hepatocellular carcinoma (HCC): KEYNOTE-240. Ann Oncol 2019;30 Suppl 4:iv135-iv6.

12. Finn RS, Ikeda M, Zhu AX, et al. Phase Ib study of lenvatinib plus pembrolizumab in patients with unresectable hepatocellular carcinoma. J Clin Oncol, 2020 (in press).

13. Kudo M, Ikeda K, Motomura K, et al. A Phase 1b Study of Lenvatinib Plus Nivolumab in Patients With Unresectable Hepatocellular Carcinoma. ASCO-GI, January 23-25, 2020, San Francisco, USA, Abstr \#513.

14. Kudo M, Finn RS, Qin S, et al. Lenvatinib versus sorafenib in first-line treatment of patients with unresectable hepatocellular carcinoma: a randomised phase 3 noninferiority trial. Lancet 2018;391:1163-73.

15. Kudo M. Pembrolizumab for the Treatment of Hepatocellular Carcinoma. Liver Cancer 2019;8:143-54.

Cite this article as: Kudo M. Combination immunotherapy with anti-VEGF/TKI for hepatocellular carcinoma: present and future perspective. HepatoBiliary Surg Nutr 202 1;10(2):241-245. doi: 10.21037/hbsn-20-707 